\title{
Breaking wave forces on a vertical pile
}

\author{
S.-J. Choi \& O. T. Gudmestad \\ Faculty of Science and Technology, University of Stavanger, Norway
}

\begin{abstract}
A 3D numerical model is developed for simulating breaking wave forces acting on a vertical pile. This model is based on the viscous and incompressible Navier-Stokes equations for a two-phase flow (water and air) model and the volume of fluid (VOF) method for the displacement of the free surface. An application based on the Cut-Cell method is employed in order to install complicated obstacles in a computational domain. The free surface elevation, dynamic pressures, and breaking wave forces are calculated by the numerical model, and the computed results show a good agreement with experimental data. The numerical model can be used to estimate breaking wave forces on a vertical pile.

Keywords: 3D numerical model, VOF method, Cut-Cell method, breaking wave force, vertical pile.
\end{abstract}

\section{Introduction}

For the design of a mono-pile structure installed on a flat bottom, Morison equation has generally been employed to determine wave forces on the structure for a given deep water wave condition. However, in the case where the monopile structure is installed in relatively shallow waters, the deep water waves may experience severely nonlinear wave deformations (i.e., breaking waves) on their propagation through steep underwater slopes, and the breaking waves generated toward the structure may give rise to serious damages to the mono-pile structure. Therefore, accurate estimation of the breaking wave forces acting on it is of great important. Until now, a conventional method such as adding an impact force, $F_{I}$, into Morison equation (see Eq. (1)) has mainly been used for estimating breaking wave forces acting on a vertical pile.

$$
F_{T}=F_{M}+F_{D}+F_{I}
$$


where, $F_{M}, F_{D}$ represent the drag and inertia force, respectively, $F_{I}$ is computed by

$$
F_{I}=\rho \cdot R \cdot C_{b}^{2} \cdot C_{s} \cdot \lambda_{c} \cdot \eta_{b}
$$

where, $\rho$ is the water density, $R$ is the radius of the pile, $C_{b}$ is the wave celerity at breaking point, $C_{s}$ is the slamming coefficient, $\lambda$ is the curling factor, and $\eta_{b}$ is the maximum surface elevation at the breaking point.

However, $C_{s}$ (slamming coefficient) and $\lambda$ (curling factor) need to be determined in advance on the basis of values presented in technical reports or additional experiments so as to estimate the breaking wave forces using the semi-empirical formula (see eq. (2)). Many experimental investigations have been carried out to estimate the slamming coefficient and the curling factor over half a century [1-6]. However, because the breaking wave impact phenomenon is extremely complicated and involves the strong non-linear effects, the results presented in the studies showed a great difference each other. Moreover, even if one of the presented results is taken for estimating the breaking wave forces, the use of the coefficients would be significantly restricted in their applications because the coefficients were determined experimentally under the influence of various external parameters, e.g., variation of water depth, diameter and inclination of pile, wave condition, and breaker type.

The use of CFD (Computational Fluid Dynamics) model is an alternative method to overcome such problems. With the rapid development of computers and computational fluid dynamics, the numerical models based on the NavierStokes equations have become popular in the research of breaking wave forces. Since the Navier-Stokes solver not only can specifically simulate strongly nonlinear characteristics of the waves occurring in the vicinity of the structure, but also can calculate the wave forces by integrating directly the pressures along the structure surface, there is no need to determine any empirical coefficients like the slamming coefficient, the curling factor, the impact duration, the drag coefficient, and the inertia coefficient for estimating the breaking wave forces on the structure.

The main objectives of this study is to propose a 3D numerical model which can estimate breaking wave forces on a vertical pile without the use of the semiempirical formula. In order to achieve the purpose of the present study, a 3D Navier-Stokes solver, based on viscous and incompressible momentum equations for a two-phase flow (water and air) model and the VOF (Volume of Fluid) method [7], is employed. The breaking wave forces on a vertical pile under plunging breakers are calculated, and the calculated results are compared with the experimental results previously undertaken by [8]. Hereby the applicability of the Navier-Stokes solver for predicting the breaking wave impact forces is verified. 


\section{Model description}

Assuming that two fluids (water and air) are viscous, incompressible, and immiscible, the fluid flow is governed by the continuity equation (equation (3)) and the modified Navier-Stokes equations (equations (4) to (6)). The free surface is governed by the volume of fluid (VOF) function in equation (7), where $F$ represent the rate of volume in a numerical cell.

$$
\begin{gathered}
\frac{\partial\left(m_{x} u\right)}{\partial x}+\frac{\partial\left(m_{y} v\right)}{\partial y}+\frac{\partial\left(m_{z} w\right)}{\partial z}=q^{*} \\
m_{v} \frac{\partial u}{\partial t}+m_{x} u \frac{\partial u}{\partial x}+m_{y} v \frac{\partial u}{\partial y}+m_{z} w \frac{\partial u}{\partial z}=-\frac{m_{v}}{\hat{\rho}} \frac{\partial p}{\partial x}+\frac{\partial}{\partial x}\left\{m_{x}\left(-\tau_{x x}+2 \hat{v} D_{x x}\right)\right\}+\frac{\partial}{\partial y}\left\{m_{y}\left(-\tau_{x y}+2 \hat{v} D_{x y}\right)\right\} \\
+\frac{\partial}{\partial z}\left\{m_{z}\left(-\tau_{x z}+2 \hat{v} D_{x z}\right)\right\}+\frac{F_{s}}{\hat{\rho}}-\frac{2 m_{x} \hat{v}}{3} \frac{\partial q^{*}}{\partial x} \\
m_{v} \frac{\partial v}{\partial t}+m_{x} u \frac{\partial v}{\partial x}+m_{v y} v \frac{\partial v}{\partial y}+m_{z} w \frac{\partial v}{\partial z}=-\frac{m_{v}}{\hat{\rho}} \frac{\partial p}{\partial y}+\frac{\partial}{\partial x}\left\{m_{x}\left(-\tau_{y x}+2 \hat{v} D_{y x}\right)\right\}+\frac{\partial}{\partial y}\left\{m_{y}\left(-\tau_{y y}+2 \hat{v} D_{y y}\right)\right\} \\
+\frac{\partial}{\partial z}\left\{m_{z}\left(-\tau_{y z}+2 \hat{v} D_{y z}\right)\right\}+\frac{F_{s}}{\hat{\rho}}-\frac{2 m_{y} \hat{v}}{3} \frac{\partial q^{*}}{\partial y} \\
m_{v} \frac{\partial w}{\partial t}+m_{x} u \frac{\partial w}{\partial x}+m_{y} v \frac{\partial w}{\partial y}+m_{z} w \frac{\partial w}{\partial z}=-\frac{m_{v}}{\hat{\rho}} \frac{\partial p}{\partial z}+\frac{\partial}{\partial x}\left\{m_{x}\left(-\tau_{z x}+2 \hat{v} D_{z x}\right)\right\}+\frac{\partial}{\partial y}\left\{m_{y}\left(-\tau_{z y}+2 \hat{v} D_{z y}\right)\right\} \\
+\frac{\partial}{\partial z}\left\{m_{z}\left(-\tau_{z z}+2 \hat{v} D_{z z}\right)\right\}+\frac{F_{s}}{\hat{\rho}}-\frac{2 m_{z} \hat{v}}{3} \frac{\partial q^{*}}{\partial z}-m_{v} g-\lambda w \\
\frac{\partial\left(m_{v} F\right)}{\partial t}+\frac{\partial\left(m_{x} u F\right)}{\partial x}+\frac{\partial\left(m_{y} v F\right)}{\partial y}+\frac{\partial\left(m_{z} w F\right)}{\partial z}=F q^{*}
\end{gathered}
$$

where, $t$ is the time, $p$ is the pressure, $u, v$, and $w$ are the velocity components in each direction, $g$ is the gravitational acceleration, $M_{v}$ is the ratio of the fractional volume open to the flow, $M_{x}, M_{y}, M_{z}$ are the ratio of the fractional area open to the flow in each direction, $\tau_{i j}$ is the turbulent stress based on the Smagorinsky SGS model, $D_{i j}$ is the stress rate tensor, $F_{s}$ is the surface tension force based on the CSF (Continuum Surface Force) model, $\lambda$ is the wave dissipation factor that equals 0 except in the added dissipation zone, $\hat{\rho}$ is the fluid density, $\hat{v}$ is the fluid kinematic molecular viscosity, and $q^{*}$ is the source term required to generate waves assigned only at the source position $\left(x=x_{s}\right)$, defined as $q^{*}=q(z, t) / \Delta x_{s}$, where $q$ is the flux density and $\Delta x_{s}$ is the mesh width at the source position.

To solve the above governing equations (equations 3 to 7 ) numerically, a fixed rectangular grid system is used in the computational domain. The variables have been staggered, which means that the pressure $(p)$, the wave source function $\left(q^{*}\right)$, and the VOF function $(F)$ are computed at the cell center, while 
the velocity components $(u, v, w)$ are computed at the center of the cell face. The continuity equation is discretized by the central difference method. As for the discretization of the Navier-Stokes equations, the forward difference method for time derivative terms, the constrained interpolation profile (CIP) method for the advection terms, and the central difference method for the non-advection terms are employed, respectively. In the computation, the velocity components $(u, v, w)$ and the pressure $P$ at the new time step are computed by using the AP-AMG (Algebraic Multigrid) solver which was developed by Allied Engineering Corporation [9], Japan. After the correct velocity components and pressure have been determined, the new free surface configuration is tracked by solving the advection equation for the VOF function.

An application based on Cut-Cell method is developed, which can easily installed complicated obstacles in the computational domain. In the application, four parameters (i.e., $M_{v}$ : ratio of fractional volume open to flow, $M_{x}, M_{y}, M_{z}$ : ratio of fractional areas open to flow in each directions), the area of the wetted surface of the structure, and the surface unit normal vectors are recorded in each cell. The governing equations (equations (3) to (7)) are formulated in terms of the computed four parameters to block portions of each cell containing the obstacle.

As for the free surface boundary conditions, because the water and the air phase are modeled as a fluid in the two-phase flow model, there is no need to apply the dynamic boundary condition at the interface between the air and the water. The dynamic boundary condition is automatically satisfied. On the other hand, the kinematic boundary condition is satisfied by tracking the VOF function. An impermeable condition (for normal velocities) and a slip condition (for tangential velocities) are imposed to treat the bottom boundary condition and the obstacle boundary condition, respectively.

\section{Application of numerical analysis}

\subsection{Computational domain}

A numerical wave tank (NWT) similar to an experimental configuration is developed. Figure 1 shows the schematic sketch of the NWT. The NWT has a length of $54.0 \mathrm{~m}$, a width of $5.0 \mathrm{~m}$, and a height of $11.4 \mathrm{~m}$. Two grids, coarse grid and fine grid, are used in the NWT. In the coarse grid, the grid size varies from a minimum of $0.1 \times 0.1 \times 0.1 \mathrm{~m}$ in the vicinity of the vertical pile to a maximum of $0.2 \times 0.2 \times 0.4 \mathrm{~m}$ far from the vertical pile, while, in the fine grid, the grid size varies from a minimum of $0.05 \times 0.04 \times 0.05 \mathrm{~m}$ to a maximum of $0.2 \times 0.2 \times 0.2 \mathrm{~m}$. The water depths are at $3.80 \mathrm{~m}$ in front of the internal wave generator $\left(d_{1}\right)$ and $1.50 \mathrm{~m}$ in the berm $\left(d_{2}\right)$, respectively. The slope of the bottom is one divided by ten. Added fictitious dissipation zones are located to the left and right sides of the computational domain with a thickness of $2 \mathrm{~L}$ ( $\mathrm{L}$ is the wave length) to absorb the wave energy. To obtain an artificial damping effect, grids in the added fictitious dissipation zones are gradually coarsened toward the outmost open boundaries. The internal wave generator, which can generate a regular wave train, is located in front of the added fictitious dissipation zone 
which was located at the left part of the computational domain. An incident wave condition (wave height, $H=1.30 \mathrm{~m}$ and wave period, $T=4.0 \mathrm{sec}$ ) is used for making breaking waves at the structural position. A vertical pile with a diameter of $0.70 \mathrm{~m}$ is located at the edge of the slope, as shown in Figure 1.19 numerical wave gauges are used to measure the water surface elevations. Moreover, 19 numerical pressure gauges are uniformly distributed along the frontline of the cylinder with a spacing of $0.2 \mathrm{~m}$. The model is run for 40 seconds (i.e., 10 wave periods).

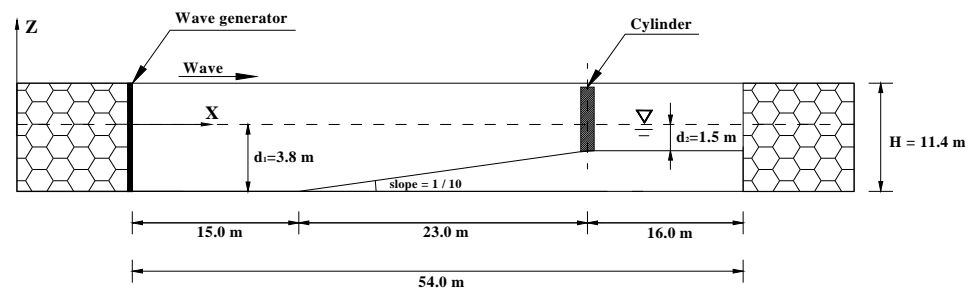

(a) Cross section of Numerical Wave Tank (NWT)

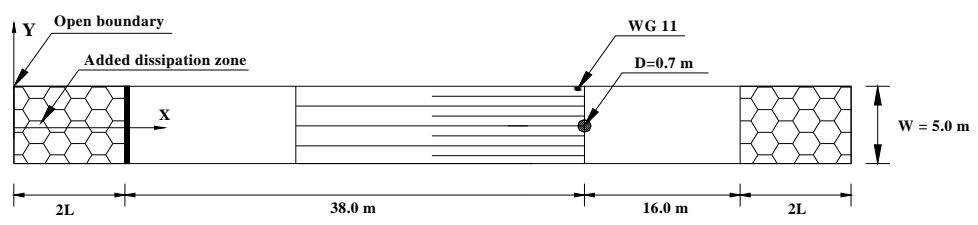

(b) Plane view of Numerical Wave Tank (NWT)

Figure 1: $\quad$ Cross section (a) and plane view (b) of the NWT (not to scale).

\subsection{Computational results and discussion}

\subsubsection{Free surface elevation}

Figure 2 shows the comparison between the calculated and the measured free surface elevation at WG11 (see Figure 2). It is clearly observed that the

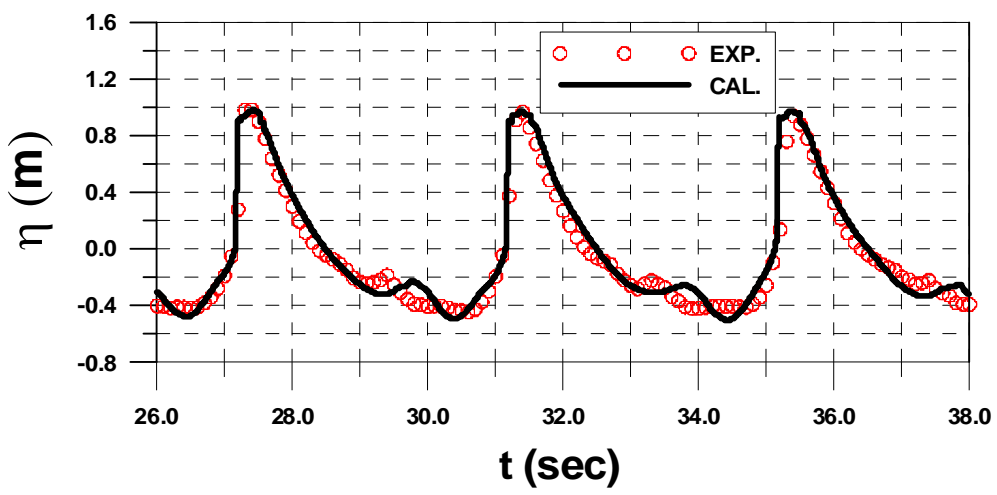

Figure 2: Comparison of free surface elevations between experimental measurements and the CFD results at WG 11 . 
numerical results agree very well with the experimental data. Especially, the numerical model can simulate relatively well the slight ripples at the wave though even though a slight phase discrepancy is observed.

\subsubsection{Dynamic pressures}

The dynamic pressures calculated by using the coarse grid, the fine grid, and the measured dynamic pressures in the experiment are compared in Figures 5 to 7. The three pressure gauges ( $\mathrm{P} 5$ to 7 ) are located at the frontline of the vertical pile (see Figure 3). In all computations with the coarse grid, the peak values are greatly underestimated compared with the measured peak values in the experiment.

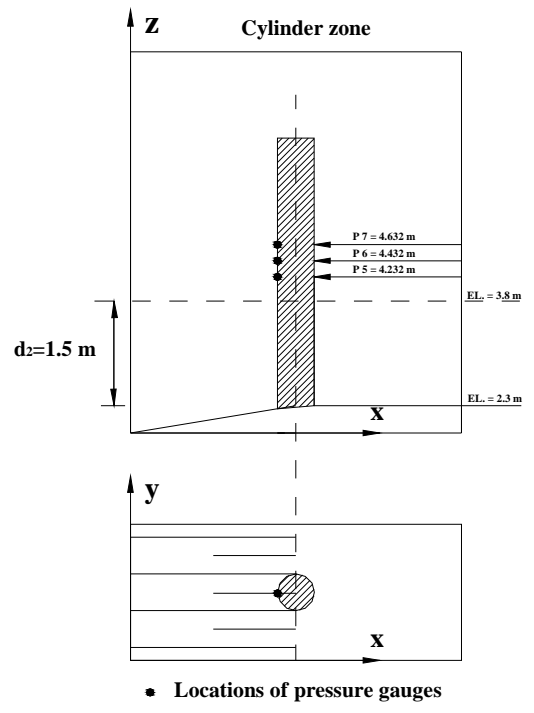

Figure 3: $\quad$ Locations of pressure gauges (pressure 5 to 7 , not to scale).

The reason for this can be explained as follows; in the experiments, the dynamic pressure is measured using a pressure transducer having a small size, which is located at position A (see Figure 4), while, in the numerical model, the dynamic pressure is computed at the centre of the cell, i.e., at position B instead of position A. In a coarse grid, the difference between these two positions is large compared to a finer grid. Hence, using a finer grid provides results which matches better with the experimental results.

In order to check the sensitivity of the grid spacing, we also present the dynamic pressures calculated using the fine grid in the same figures. On the whole, the peak values calculated using the fine grid show a reasonable agreement with measured peak values. From the comparisons, it can be concluded that the accuracy of the simulation results depend greatly on the cell resolution. Meanwhile, in P 5 and P 6 (see Figures 5 and 6), the computed risetimes are significantly smaller than the measured rise-times even though the 


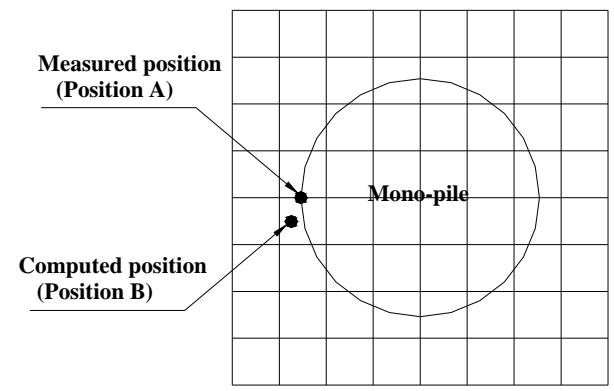

Figure 4: Locations of measured position (position A) and computed position (position B).

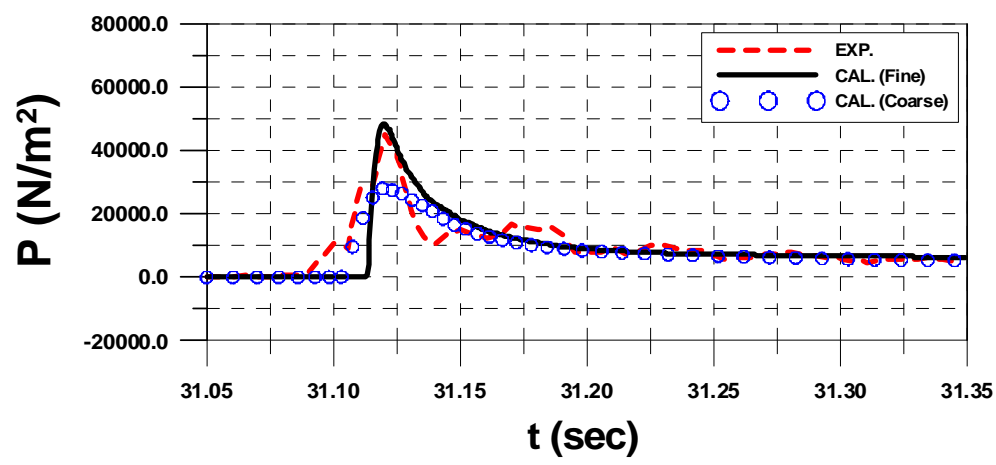

Figure 5: Comparison of dynamic pressures using the coarse grid, dynamic pressures using the fine grid, and the measured dynamic pressures at P 5.

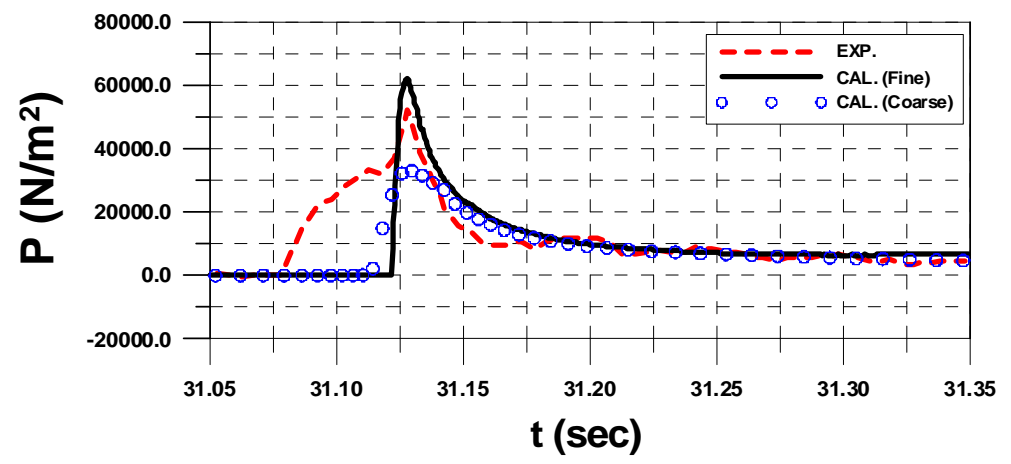

Figure 6: Comparison of dynamic pressures using the coarse grid, dynamic pressures using the fine grid, and the measured dynamic pressures at P 6. 
computed fall-times shows a reasonable agreement with the measured fall-times. The reason for this is not clear at present and should be investigated further. However, it should be noticed that, in $\mathrm{P} 7$, the rise-time and the fall-time are reproduced very well in the numerical result (see Figure 7).

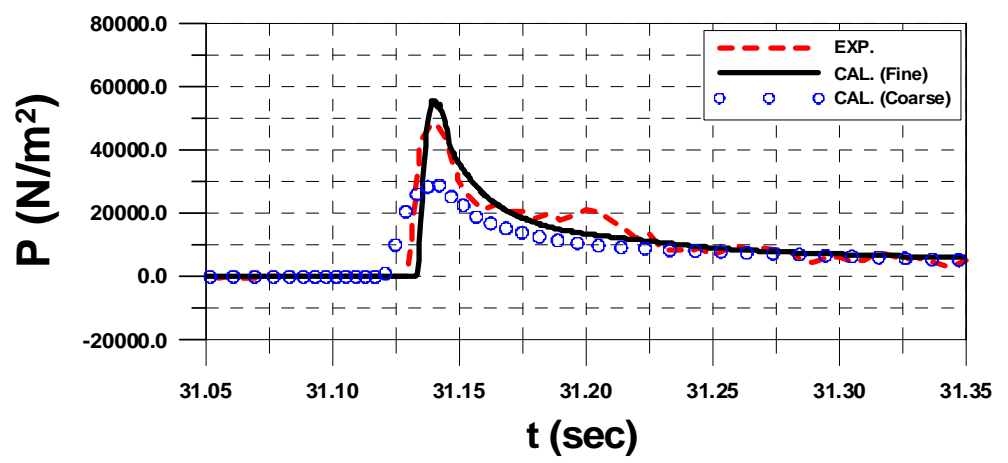

Figure 7: Comparison of dynamic pressures using the coarse grid, dynamic pressures using the fine grid, and the measured dynamic pressures at P 7.

\subsubsection{Breaking wave forces on a vertical pile}

It should be mentioned here that the pile is modelled as a rigid object in the numerical analysis. On the other hand, in the experiment, a structure must move in order to induce sufficient strain in the force transducer, or in other words, it must be flexible. The total force time series data obtained from the experiment, when converted into frequency domain, shows a peak that corresponds to the natural frequency of the structure $(17.1 \mathrm{~Hz})$. This is expected, since the natural frequency of the structure is close to the impact force duration. Hence, in order to compare with the numerical results, the total impact force data, obtained from the experiment, must be filtered to remove the amplifying effect due to the structure's vibration. An approach similar to that described in [10] is adopted here.

Unfiltered wave force data, filtered wave force data, and numerical results are compared in Figure 8. It is clearly observed that the filtered data are in good agreement with the numerical results, while the unfiltered data shows a larger response at the beginning of the impact, and subsequent oscillations due to the structure's vibration in its natural frequency. The large difference between the peak responses in the unfiltered and the filtered data shows the prominent effect of the dynamic amplification. The dynamic amplification factor (DAF) in the experimental data can be expressed, as shown in Equation 8.

$$
D A F=\frac{F_{\text {Unfiltered }}}{F_{\text {Filtered }}}
$$

The DAF is estimated here as 1.79. This shows the importance of studying the shape and the duration of the breaking wave induced impulsive load, which, 
along with the structure's natural frequency, governs the DAF in the structure's response. For example, in a jacket type foundation of an offshore wind turbine, some of the members may have their local natural frequency in the vicinity of the impact duration during a breaking wave; this may cause large local vibrations in those members.

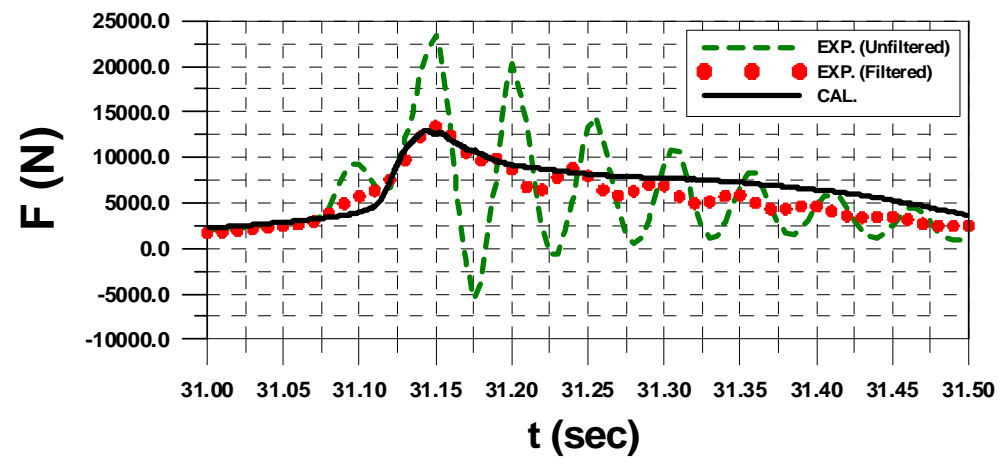

Figure 8: Comparison of unfiltered wave force data, filtered wave force data, and breaking wave forces calculated by the CFD model.

\section{Conclusions}

A 3D numerical model is employed to study the breaking wave phenomenon in front of a vertical pile. The free surface elevation, the dynamic pressures, and the impact force acting on the pile are calculated, and the results are compared with the experimental data obtained by [8]. The major conclusions, based on the numerical results, can be summed up as follows;

1. The numerical model shows good performance to model the breaking wave phenomenon, and to produce reliable outputs (free surface elevation, dynamic pressure, and impact forces); this can be a good replacement to the expensive experiments in future. However, more comparative studies are required to increase the confidence level.

2. It is clearly observed that the accuracy of estimating the breaking wave impact pressure depends on the grid resolution. The dynamic pressures calculated by using a finer grid show a better agreement with experimental data compared to a coarse grid.

3. The computed breaking wave forces, and the experimental data filtered for the natural frequency of the structure are found to be in good agreement with each other. This, in fact, indirectly shows that the structure's response can greatly be influenced by the time duration and the shape of the impulsive load in a breaking wave.

4. The proposed numerical model can be used to estimate the breaking wave forces on structures, e.g., mono-pile, jacket foundation etc., without using the semi-empirical equation. 


\section{Acknowledgements}

The research was supported by a grant ("stipendiat-TN-2008”) from the Research Council of Norway. The physical model test data was provided by Lisham Bonakdar and Professor Hocine Oumeraci, TU Braunschweig, Germany. The AP-AMG solver for solving Poisson Pressure Equation (PPE) was provided by Chihiro Iwamura, Allied Engineering Corporation, Japan.

\section{References}

[1] Goda, Y., Haranaka, S., and Kitahata, M., Study of impulsive breaking wave forces on piles, Report of port and harbour research institute, 5 (6), pp. 1-30, 1966.

[2] Honda, T. and Mitsuyasu, H., Experimental study of breaking wave force on a vertical circular cylinder, Coastal Engineering in Japan, 17, pp. 59-70, 1974.

[3] Sawaragi, T. and Nochino, M., Impact forces of nearly breaking waves on a vertical circular cylinder, Coastal Engineering in Japan, 27, pp. 249-263, 1984.

[4] Tanimoto, K., Takahashi, S., Kaneko, T. and Shiota, K., Impulsive breaking wave forces on an inclined pile exerted by random waves, Proc. of the $20^{\text {th }}$ Inter. Conf. on Coastal Engineering, pp. 2288-2302, 1986.

[5] Wienke, J., and Oumeraci H., Breaking wave impact on a vertical and inclined slender pile-theoretical and large-scale model investigations, Coastal Engineering, 52, pp. 435-462, 2005.

[6] Arntsen, Ø., Ros, X. and Tørum, A., Impact forces on a vertical pile from plunging breaking waves, Coastal Structures 2011, Yokohama, 2011.

[7] Hirt, C.W. and Nichols, B. D., Volume of fluid method for the dynamics of free boundaries, Journal of Computational Physics, 39 (1), pp. 201-225, 1981.

[8] Kai Irschik, Uwe Sparboom, and Hocine Oumeraci., Breaking wave characteristics for the loading of a slender pile, Proc. of the $28^{\text {th }}$ Int. Conf. on Coastal Engineering, pp. 1341-1352, 2002.

[9] Allied Engineering, User's Manual for Advanced Parallel AMG Version 1.3, Tokyo, 2011.

[10] Subrata Kumar Chakrabarti., The theory and practice of hydrodynamics and vibration. Advanced series on Ocean Engineering - Volume 20, Would Scientific, 2002. 